\title{
(อ) OPEN ACCESS \\ Psychological, social and contextual factors across recovery stages following a sport-related knee injury: a scoping review
}

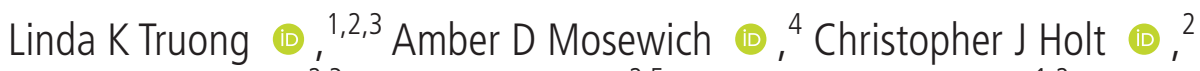 \\ Christina Y Le (10), 2,3 Maxi Miciak (1) , 2,5 Jackie L Whittaker (1) 1,2
}

- Additional material is

published online only. To view please visit the journal online (http://dx.doi.org/10.1136/ bjsports-2019-101206).

${ }^{1}$ Faculty of Medicine, Department of Physical Therapy, \#223, 212 Friedman Building 2177 Westbrook Mall, V6T 1Z3, The University of British Columbia, Vancouver, British Columbia, Canada ${ }^{2}$ Faculty of Rehabilitation Medicine, Department of Physical Therapy, University of Alberta, Edmonton, Alberta, Canada Clinic, Edmonton, Alberta, Canada

${ }^{4}$ Faculty of Kinesiology, Sport, and Recreation, University of Alberta, Edmonton, Alberta, Canada

${ }^{5}$ Alberta Innovates, Edmonton, Alberta, Canada

\section{Correspondence to} Linda K Truong, Faculty of Medicine, Department of Physical Therapy, \#223, 212 Friedman Building 2177 Westbrook Mall, V6T 1Z3, The University of British Columbia, Vancouver, Canada; Iltruong@ualberta.ca

Accepted 26 January 2020 Published Online First 14 February 2020
${ }^{3}$ Glen Sather Sports Medicine

\section{ABSTRACT}

Objective To explore the role of psychological, social and contextual factors across the recovery stages (ie, acute, rehabilitation or return to sport (RTS)) following a traumatic time-loss sport-related knee injury.

Material and methods This review followed the Preferred Reporting Items for Systematic Reviews and Meta-Analyses Extension for Scoping Reviews and Arksey and O'Malley framework. Six databases were searched using predetermined search terms. Included studies consisted of original data written in English that identified or described a psychological, social or contextual factor related to recovery after a traumatic time-loss sport-related knee injury. Two authors independently conducted title-abstract and full-text reviews. Study quality was assessed using the Mixed Methods Appraisal Tool. Thematic analysis was undertaken.

Results Of 7289 records, 77 studies representing 5540 participants (37\% women, 84\% anterior cruciate ligament tears, aged 14-60 years) were included. Psychological factors were investigated across all studies, while social and contextual factors were assessed in $39 \%$ and $21 \%$ of included studies, respectively. A cross-cutting concept of individualisation was present across four psychological (barriers to progress, active coping, independence and recovery expectations), two social (social support and engagement in care) and two contextual (environmental influences and sport culture) themes. Athletes report multiple barriers to recovery and valued their autonomy, having an active role in their recovery and diverse social support.

Conclusion Diverse psychological, social and contextual factors are present and influence all stages of recovery following a traumatic sport-related knee injury. A better understanding of these factors at the time of injury and throughout rehabilitation could assist with optimising injury management, promoting RTS, and long-term health-related quality-of-life.

\section{Check for updates}

(C) Author(s) (or their employer(s)) 2020. Re-use permitted under CC BY-NC. No commercial re-use. See rights and permissions. Published by BMJ.

To cite: Truong LK,

Mosewich AD, Holt CJ,

et al. Br I Sports Med

2020;54:1149-1156.

\section{BACKGROUND}

Up to $40 \%$ of reported time-loss sport injuries involve knee trauma. ${ }^{12}$ Despite best efforts, many athletes do not return to sport (RTS) following a traumatic knee injury ${ }^{34}$ and/or develop early-onset post-traumatic osteoarthritis (PTOA), irrespective of management approach. ${ }^{5-7}$ Traditionally, injury recovery predominantly focused on resolving physical impairments. However, improved physical outcomes are not always associated with return to physical activity, ${ }^{8}$ long-term satisfaction ${ }^{9}$ or favourable health-related quality-of-life (HRQoL), ${ }^{10}$ suggesting that additional 'non-physical factors' may mediate recovery. These non-physical factors likely encompass a broad range of psychological, social and contextual domains. ${ }^{11}$

Psychological factors that influence recovery from a traumatic, time-loss, sport-related knee injury include cognitive (eg, perceptions), behavioural (eg, adherence) and affective (eg, moods) responses associated with an individual's experience of the injury, rehabilitation, surgery and RTS. ${ }^{11}{ }^{12}$ For example, fear of re-injury and poor psychological readiness for RTS in individuals with an anterior cruciate ligament (ACL) tear negatively influence activity levels and RTS. ${ }^{13-15}$ Similarly, a recent systematic review highlighted the association between greater preoperative self-efficacy (ie, belief that one is capable of executing a behaviour in a specific context) ${ }^{16}$ and less future knee pain and favourable functional and RTS outcomes following an ACL reconstruction (ACLR). ${ }^{17}$ Social factors that influence recovery in this population include the social networks (eg, family, sport, therapy) and social exchanges (eg, relationships, support) that influence, and are influenced by an individual's injury experience. ${ }^{18} 19$ Specifically, effective communication and a strong patient-therapist (therapeutic) alliance have been shown to be associated with improved rehabilitation outcomes following a musculoskeletal injury. ${ }^{2021}$ Contextual factors that influence an individual's injury experience include the structural and institutional systems that they are embedded in (eg, physical or sport environment, ethnicity, socioeconomic status). ${ }^{1922}$ For instance, cultural perceptions shaped by one's ethnicity or societal influences (eg, community views about disability) could impact beliefs, recovery expectations, rehabilitation satisfaction, and HRQoL in individuals with chronic low back pain. 2324

A holistic approach that considers physical, psychological, social and contextual factors was recommended to optimise recovery in a recent consensus statement on sport-related injuries. ${ }^{25}$ Yet, evidence about the psychological, social and contextual factors influencing recovery following a timeloss sport-related knee injury is lacking, making clinical application of these recommendations challenging. Although previous reviews ${ }^{13} 15$ have broadly summarised psychosocial factors related to sport injury outcomes, no review has looked at psychological and social factors as distinct domains or considered contextual factors after a time-loss 
traumatic knee injury. Unlike a systematic review, which aims to synthesise evidence from multiple studies to answer a specific research question, ${ }^{26}$ a scoping review aims to map the breadth and depth of the current evidence around a particular topic that is dispersed across disciplines and heterogeneous study designs. ${ }^{27-29}$ The aim of this scoping review is to consolidate and examine the evidence related to the role of psychological, social and contextual factors across the recovery stages (ie, acute, rehabilitation or RTS) following a traumatic time-loss sport-related knee injury to identify key themes and knowledge gaps.

\section{METHODS}

This review was conducted and reported according to the Preferred Reporting Items for Systematic Reviews and MetaAnalyses Extension for Scoping Reviews. ${ }^{30}$ We followed the methodological framework of Arksey and O'Malley (2005) with refinements proposed by Levac et al (2010) and the Johanna Briggs Institute (2015). ${ }^{27-29}$ At the initiation of this review, there was no database to register a priori scoping review strategies.

\section{Study team}

To facilitate robust and clinically relevant review findings, ${ }^{29}$ the study team included individuals with expertise in evidence synthesis, quantitative and qualitative research methodology, clinical epidemiology, sport and exercise psychology, clinical therapeutic relationships, and sport and knee injury rehabilitation.

\section{Data sources and search}

Relevant studies were identified by searching six online databases (ie, MEDLINE, PsycINFO, CINAHL, SportDiscus, SCOPUS and ProQuest) from inception to May 2018, selected based on their relevance to the research topic. Keywords and constructs (ie, MeSH, Boolean phrases) used to execute each search were developed a priori from a preliminary search, conceptual papers, ${ }^{11} 12182231$ search strategies from relevant systematic reviews, ${ }^{1314}$ and in consultation with team members and a health sciences librarian scientist. A list of search terms is provided in table 1, and the full search strategies for all databases are found in online supplementary file 1 . To ensure that the search strategy was capturing relevant records, an iterative process involving team meetings to refine search terms and resolve challenges was used. ${ }^{27} 29$ One specific challenge was operationalising 'psychological', 'social' and 'contextual'. In the end, search terms were refined to include examples of each. All searches were limited to the English language, conducted by the lead author, and organised using the reference management software EndNote X8.1.

\section{Study screening}

After accounting for duplication, the titles and corresponding abstracts of all returned records were independently reviewed by two raters, blinded to record author(s) and journal title. Prior to the title and abstract reviews, all raters independently screened a random sample of 120 titles and abstracts to assess the applicability of the exclusion criteria and inter-rater agreement and Cohen's kappa $(K)$ with the senior author. All reviewers reached acceptable agreement $(95 \%-98 \%, K=0.75-0.90)$. Finally, two independent raters performed full-text screening to determine final study selection. Consensus was reached on disagreements, first between raters and if required with the senior author. A review of the reference list of included studies, relevant systematic or literature reviews, and clinical guidelines was used to identify additional relevant records.

\section{Study selection}

Studies were included if they identified, described or assessed a psychological, social or contextual factor during the acute, rehabilitation or RTS stages of recovery following a traumatic time-loss knee injury resulting from or interfering with sport participation. See table 2 for definitions and table 3 for inclusion and exclusion criteria.

\section{Data extraction}

Data extracted from each study included the following: study year, design, location and aim; sport and sport participation level; sample age and size; injury type (eg, ACL, ACLR, patella dislocation); instrument(s) or approach(s) used to assess psychological, social and/or contextual factor; recovery stage (ie, acute, rehabilitation or RTS); and study result (ie, identification or description of psychological, social and/or contextual factor during the acute, rehabilitation or RTS stages of recovery). For studies that asked participants to reflect on past experiences, the assigned recovery stage was categorised based on the period being assessed.

Data extraction was completed by the lead author using a customised form. Prior to data extraction, the performance of the form was assessed by comparing data extracted by two independent raters across a purposive sample of 12 studies of various designs to ensure accurate and relevant data were extracted across different study designs.

\section{Data synthesis}

Individual psychological, social and contextual factors were identified across studies. Factors were operationalised based on consultation with the study team and relevant sport injury or

\begin{tabular}{|c|c|}
\hline Construct & Keywords \\
\hline $\begin{array}{l}\text { Population: } \\
\text { (sport participants with a lower body injury) }\end{array}$ & 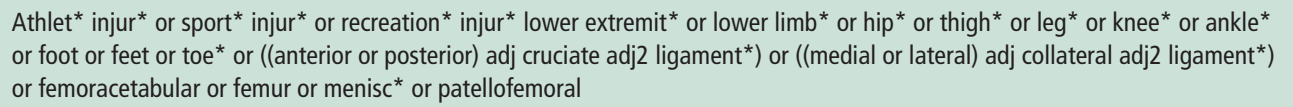 \\
\hline $\begin{array}{l}\text { Injury type: } \\
\text { (musculoskeletal) }\end{array}$ & tear* $^{*}$ or rupture* or ligament* or sprain* or strain* or dislocation* \\
\hline $\begin{array}{l}\text { Non-physical factor: (psychological, social, } \\
\text { contextual) }\end{array}$ & 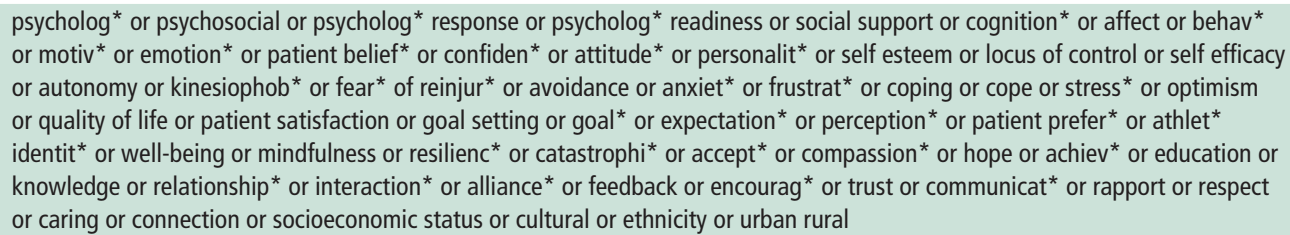 \\
\hline
\end{tabular}

*initial search strategy targeted all traumatic sport-related lower extremity injuries. 
Table 2 Operationalised definitions

\begin{tabular}{|c|c|}
\hline Term & Definition \\
\hline Psychological factor & Cognitive, behavioural or affective responses associated with an individual's experience of injury, rehabilitation, surgery and RTS ${ }^{11}$ \\
\hline Social factor & Social networks and social exchanges that shape an individual's experience of injury, rehabilitation, surgery and RTS ${ }^{1832}$ \\
\hline Contextual factor & $\begin{array}{l}\text { Structural and institutional systems in which an individual is embedded that influence their experience of injury, rehabilitation, surgery and } \\
\text { RTS }^{2231}\end{array}$ \\
\hline Acute recovery stage & The time period immediately post-injury and/or pre-surgery or post-surgery \\
\hline Rehabilitation recovery stage & The time period between the acute and RTS stage that is focused on restoring physical and psychological function \\
\hline RTS recovery stage & The time period when an injured athlete is engaged in a graduated RTS programme or have returned to sport and/or physical activity \\
\hline Traumatic time-loss knee injury & $\begin{array}{l}\text { Any trauma to the knee (joint, ligament, muscles) caused by a transfer of energy that exceeded the body's ability to maintain its structural } \\
\text { and/or functional integrity and resulted in a player missing at least one training session (practice) or match play }\end{array}$ \\
\hline Sport participation & $\begin{array}{l}\text { Participation in any level (recreation, competitive or professional) of organised sport at time of injury }{ }^{130} \\
\text { Recreational=sport participation primarily for fun and entertainment } \\
\text { Competitive=amateur sport participation at a high competitive level (eg, varsity or collegiate level) } \\
\text { Professional=elite sport participation at the highest level of a sport (eg, national, international, professional level athletes) with } \\
\text { monetary support (eg, salary/carding, stipends, sponsorships) }\end{array}$ \\
\hline
\end{tabular}

RTS, return to sport.

conceptual papers. $^{11} 12182232$ An inductive thematic analysis ${ }^{33}$ was used to identify patterns, summarise consistent findings across studies and generate common themes. The Biopsychosocial Model ${ }^{11}$ and WHO HRQoL framework ${ }^{34}$ were used to categorise individual factors and themes into psychological, social and contextual domains. These conceptual models were used to facilitate translation of the findings to patients, healthcare providers and other stakeholders and assist in the identification of knowledge gaps. Regular study team meetings were held to discuss and agree on emerging themes and interpretations.

\section{Quality assessment}

The methodological quality of included studies was assessed by two independent raters using the Mixed Methods Appraisal Tool (MMAT) 2018 Version (online supplementary file 2). ${ }^{35}$ The MMAT assesses five different methodological categories, allowing the tool to be used across various study designs and is reliable, valid and efficient. ${ }^{35-37}$ The level of evidence represented by each record was determined with a modified version of the Oxford Centre of Evidence Based Medicine (OCEBM) 2009 model (online supplementary file 3). Discrepancies in the MMAT scoring or OCEBM categorisation were resolved by consensus between the raters and as needed with the senior author.

\section{Rigor}

The iterative nature of a scoping review allows for refinement of exclusion and inclusion criteria to ensure an adequate yet feasible scope of relevant evidence. ${ }^{27} 29$ The initial scope of this

\begin{tabular}{ll}
\hline Table 3 & \multicolumn{1}{c}{ Study exclusion and inclusion criteria } \\
\hline Inclusion criteria & Exclusion criteria \\
\hline $\begin{array}{l}\text { English language } \\
\text { Human participants }\end{array}$ & $\begin{array}{l}\text { Not English language } \\
\text { Animal models or cadavers }\end{array}$ \\
\hline $\begin{array}{l}\text { Primary study design with } \\
\text { original data }\end{array}$ & $\begin{array}{l}\text { Not primary or original data (eg, conference } \\
\text { proceedings or abstracts, editorials, commentaries, } \\
\text { opinion-based papers or reviews) }\end{array}$ \\
\hline $\begin{array}{l}\text { Traumatic time-loss knee injury } \\
\text { Do not involve a traumatic time-loss knee joint } \\
\text { injury }\end{array}$ \\
$\begin{array}{l}\text { Describes a psychological, } \\
\text { social or contextual factor } \\
\text { during recovery (acute, } \\
\text { rehabilitation or RTS) }\end{array}$ & $\begin{array}{l}\text { Does not describe a psychological, social, contextual } \\
\text { factor during recovery (eg, injury prevention, } \\
\text { epidemiology or surgical technique studies) }\end{array}$ \\
\hline
\end{tabular}

RTS, return to sport. review included grey literature (ie, PhD theses) and all sportrelated lower extremity injuries. Given the small number of records remaining after title/abstract screening that assessed non-knee injuries $(n=5)$ or grey literature $(n=18)$, a decision was made to focus on published peer-reviewed studies and knee injuries. Of the 16 grey literature records pertaining to the knee, the findings of three were included as peer-reviewed papers and the remaining were re-visited to ensure no theme or construct was missed.

\section{RESULTS}

\section{Identification of studies}

An overview of the study identification process is provided in figure 1. Of 7289 potential records, 4746 unique records underwent title/abstract screening, 293 were reviewed in full, and 77 studies were included.

\section{Study characteristics}

In all, $54(70 \%)$ studies were quantitative (involving longitudinal $(30 \%),{ }^{38-63}$ cross-sectional $(27 \%)^{64-84}$ or case series $(5 \%)^{85-88}$ designs), three (4\%) included an intervention, ${ }^{89-91} 18(23 \%)$ studies were qualitative ${ }^{92-109}$ (consisting of seven (39\%) descriptive, five $(28 \%)$ case or narrative, three $(17 \%)$ grounded theory, and three (17\%) phenomenology approaches) and five (6\%) mixed methods designs. ${ }^{110-114}$ Full details of study characteristics are summarised in online supplementary file 4 .

Studies represented data from 5540 participants $(\mathrm{men}=2986$, women $=2030$ ) ranging in age from 14 to 60 years from 14 countries. Across studies, participants represented a variety of sport participation levels. Most studies investigated individuals 18 years of age or older (77\%), with only two studies investigating adolescence. In all, 65 (84\%) of studies involved individuals following ACL injury or ACLR, with the remaining investigating individuals with a patella dislocation, meniscectomy or other ligament injury.

\section{Psychological, social and contextual factors and recovery stage}

Table 4 provides the summary and definition of the psychological, social and contextual factors identified across studies. Psychological factors were reported in all studies, while only $39 \%$ and $21 \%$ of included studies reported on social and contextual factors, respectively. Most studies were performed during 


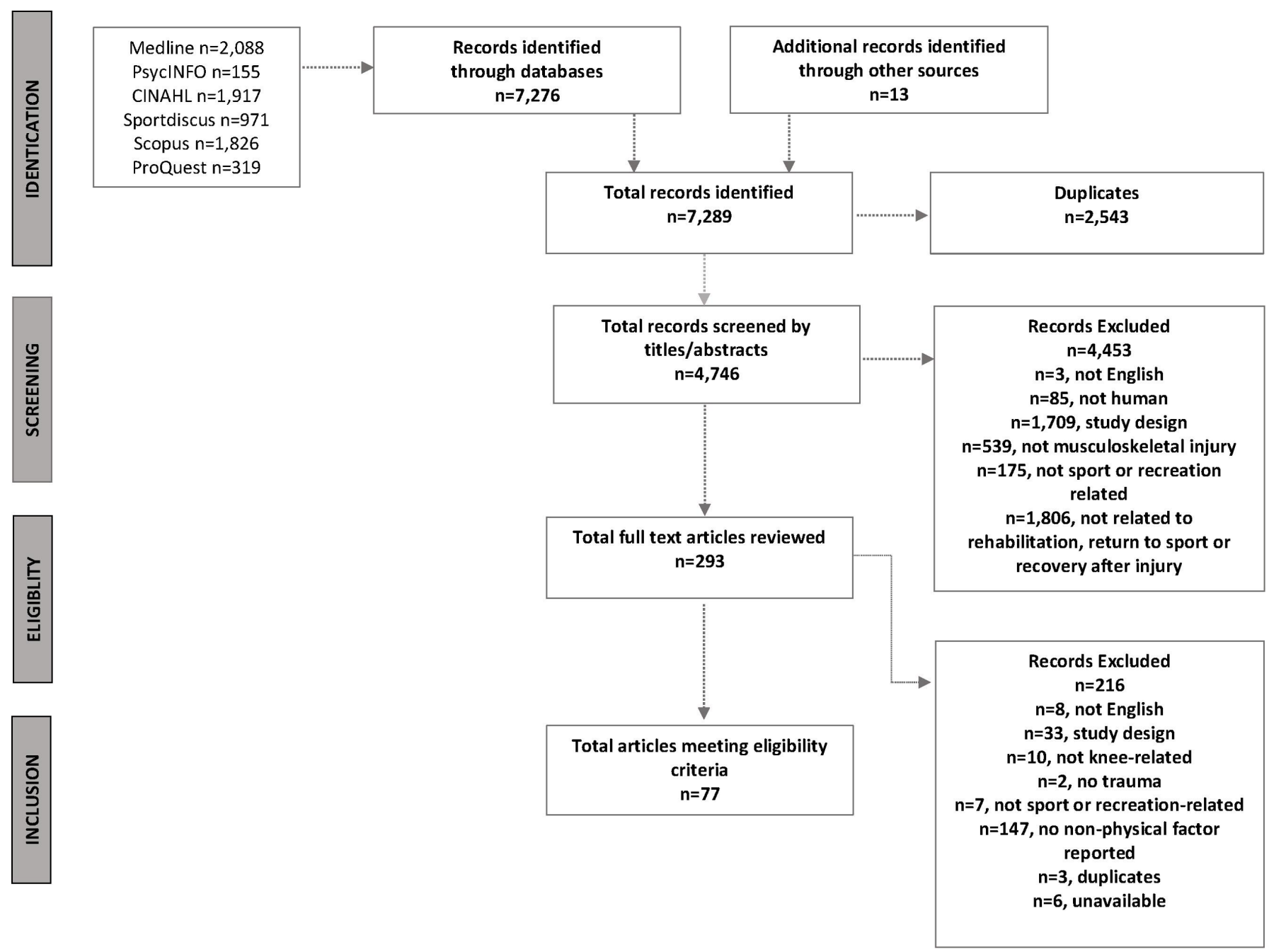

Figure 1 Preferred Reporting Items for Systematic Reviews and Meta-Analyses Extension for Scoping Reviews flow chart.

rehabilitation (47\%) or RTS (37\%) recovery stage, with only $15 \%$ of included studies addressing the acute stage.

\section{Data synthesis}

Higher-level themes that represented consistent findings and patterns across studies are presented in figure 2. These themes were further categorised into psychological, social or contextual domains resulting in four psychological, two social and two contextual themes with one cross-cutting concept.

\section{Psychological domain themes}

\section{Barriers to progress}

Athletes experience a variety of barriers to progress during recovery from a knee injury. While fear was most commonly reported, other emotional barriers such as frustration and anxiety ${ }^{6892-94100101105106108112113}$ were evident throughout all recovery stages. ${ }^{41} 5768839396100110$ Fear was most commonly seen as a major barrier during RTS. 6165737678829295105106110113114 However, only $20 \%-45 \%$ of athletes stated fear as the main reason for not returning to sport ${ }^{73} 7678113$ with psychological readiness, ${ }^{48} 648485$ knee and sport confidence, ${ }^{92} 94114$ and motivation $^{5458}$ identified as other barriers for RTS. Beyond recovery, fear of re-injury was associated with ACL re-injury within 2-5 years ${ }^{53} 88$ and reduced activity levels 3-20years following ACLR. ${ }^{4795113}$ Across studies, early recognition and acknowledgement of negative emotions were important for recovery. ${ }^{93} 102110$

\section{Active coping}

Injured athletes value playing an active role in their recovery. Immediately after injury, athletes reported wanting strategies that help them understand their injury and diagnosis, ${ }^{94} 103110$ manage emotions $^{8793106}$ and deal with athletic identity loss. ${ }^{96-98} 106108109$ Approaches to managing recovery setbacks (eg, flare-ups or re-injuries) $)^{105112}$ and problem-based coping tactics during RTS ${ }^{92}$ were also important. There was a continued need for active coping strategies beyond RTS to assist in accepting the long-term consequences of knee injury, ${ }^{9495}$ improve knee confidence ${ }^{75}$ and transition to a life outside of sport. ${ }^{98} 107$ An absence of coping strategies was associated with being unprepared for the mental demands of recovery, ${ }^{97}$ depressed feelings,${ }^{108}$ failed RTS ${ }^{102}$ and maladaptive behaviours such as substance abuse or catastrophising pain. ${ }^{40}$ In contrast, providing active coping strategies was associated with greater motivation, 99101102110111 resilient behaviour, ${ }^{99112}$ and adherence to rehabilitation. ${ }^{93} 104$

\section{Independence}

Across studies, injured athletes reported a desire to develop or preserve their independence and have control over their rehabilitation. ${ }^{42} 5660676970939799$ 101-103112 Developing greater 
Table 4 Categorisation and definitions of identified psychological, social and contextual factors and themes

\begin{tabular}{|c|c|c|c|}
\hline Theme & Factor & Definition & No. of studies \\
\hline Psychological domain & Psychological factor & & 77 \\
\hline \multirow[t]{4}{*}{ Barriers to progress } & Fear/anxiety ${ }^{131}$ & $\begin{array}{l}\text { Fear* is a biological mechanism associated with definite danger (eg, fear of re-injury), whereas anxiety is } \\
\text { associated with anticipation or uncertainty from previous experience (eg, re-injury anxiety) }\end{array}$ & 29 \\
\hline & Other emotions ${ }^{32}$ & $\begin{array}{l}\text { The way one feels after an event (eg, injury) and can consist of feelings and/or mood disturbances (eg, } \\
\text { frustration, anger, depression) }\end{array}$ & 24 \\
\hline & Psychological readiness ${ }^{32}$ & $\begin{array}{l}\text { A state of mental preparedness. In the context of RTS, this includes an appraisal of one's health status and } \\
\text { risks (perceived or actual) associated with sport participation }\end{array}$ & 8 \\
\hline & Sport-confidence $^{132}$ & The belief or degree of certainty individuals possess about their ability to be successful in sport & 6 \\
\hline \multirow[t]{4}{*}{ Active coping } & Coping ${ }^{133}$ & $\begin{array}{l}\text { Cognitive and behavioural efforts used to manage demands that are appraised as taxing or exceeding one's } \\
\text { resources }\end{array}$ & 19 \\
\hline & Identity ${ }^{134}$ & An internal, self-constructed, dynamic organisation of drives, abilities, beliefs, and individual history & 13 \\
\hline & Catastrophising pain ${ }^{24}$ & A negative appraisal of pain that may represent ineffective or inappropriate pain coping strategy & 4 \\
\hline & Resilience ${ }^{135}$ & $\begin{array}{l}\text { The ability to use personal qualities to withstand pressure, 'bounce back', and persist during stressful or } \\
\text { adverse encounters (eg, injury) }\end{array}$ & 2 \\
\hline \multirow[t]{3}{*}{ Independence } & Self-efficacy ${ }^{16}$ & $\begin{array}{l}\text { A situation-specific form of confidence that involves self-assessment or judgement of one's capability to } \\
\text { execute behaviours to attain an outcome }\end{array}$ & 12 \\
\hline & Motivation ${ }^{115}$ & All aspects of activation and intention on behaviour, including energy, direction, persistence and equifinality & 10 \\
\hline & Autonomy ${ }^{115}$ & $\begin{array}{l}\text { An internal perceived locus of causality or degree to which individuals desire to control life events or } \\
\text { behaviour }\end{array}$ & 4 \\
\hline \multirow[t]{3}{*}{ Recovery expectations } & Recovery expectation ${ }^{127}$ & A belief that some anticipated future outcome is likely & 12 \\
\hline & Perceptions ${ }^{136}$ & How one views or perceives their current situation or injury & 8 \\
\hline & Beliefs $^{136}$ & A dispositional mindset that may or may not manifest either in consciousness or behaviour & 5 \\
\hline Social domain & Social factor & & 30 \\
\hline Social Support & Social support ${ }^{137}$ & $\begin{array}{l}\text { An exchange of resources or social interactions between individuals comprising of emotional, informational } \\
\text { and tangible support }\end{array}$ & 29 \\
\hline \multirow[t]{2}{*}{ Engagement in care } & Therapeutic alliance ${ }^{138}$ & $\begin{array}{l}\text { A working relationship between a healthcare provider and patient that involves agreement on treatment } \\
\text { goals, tasks, and an affective bond (eg, trust) }\end{array}$ & 7 \\
\hline & Shared decision-making ${ }^{125}$ & $\begin{array}{l}\text { A process whereby healthcare providers and patients work together to make healthcare choices based on } \\
\text { best available evidence and patient's values }\end{array}$ & 4 \\
\hline Contextual domain & Contextual factor & & 16 \\
\hline Environmental influences & Environment $^{139}$ & $\begin{array}{l}\text { The atmosphere or setting of a situation which is created by a combination of external (eg, healthcare } \\
\text { provider) factors and/or one's individual context }\end{array}$ & 12 \\
\hline Sport culture & Sport culture $^{139}$ & The environment or ethos of a sport & 4 \\
\hline
\end{tabular}

*Given that fear has been heavily investigated in this population, we chose to separate it from 'other emotions.' Fear and anxiety (fear of re-injury/re-injury anxiety) were grouped together as these two constructs were often used interchangeably across studies.

RTS, return to sport.

independenceby promotingself-motivation, ${ }^{54646770768587101104-106}$ confidence, $^{78} 929498100102105108113114$ self-efficacy, ${ }^{60} 8199$ and autonomy around decisions for RTS and future activity choices $^{42} 6997103104$ were reported to positively influence either rehabilitation or RTS outcomes. Furthermore, fostering psychological readiness for RTS promoted independence, self-awareness on readiness to RTS and greater confidence on RTS. 394864848592100102103109

\section{Recovery expectations}

Establishing realistic expectations for rehabilitation or RTS $^{9798103106108}$ and long-term recovery ${ }^{66109}$ was important to athletes recovering from knee injuries. However, several studies identified unrealistic expectations about the length of recovery by athletes requiring surgery. ${ }^{55727983}$ Not meeting recovery expectations was associated with negative emotions (eg, frustration) and a loss of confidence during rehabilitation. ${ }^{97} 98112$

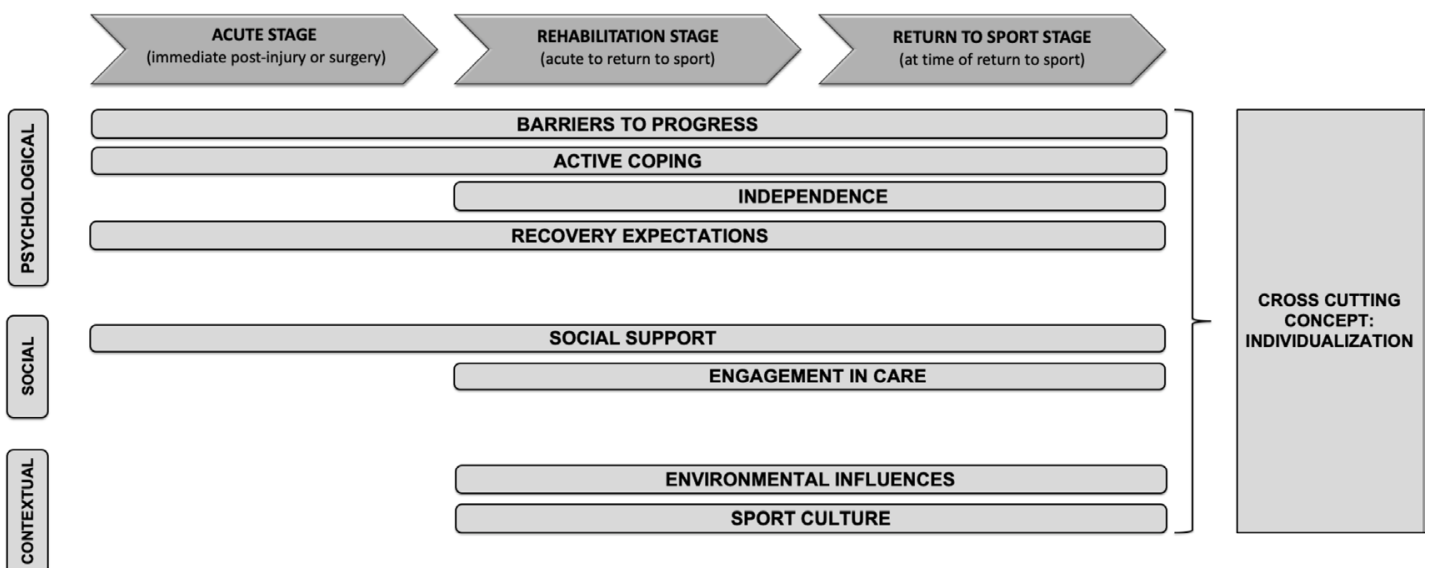

Figure 2 Representation of psychological, social and contextual themes by recovery stage. 
Interestingly, recovery expectations were influenced by interactions with healthcare providers, ${ }^{98} 106$ high-level role models, other players and coaches, ${ }^{98} 105$ prior perceptions, ${ }^{87}$ and/or beliefs. ${ }^{6883}$

\section{Social domain themes}

\section{Social support}

The exchange of resources such as informational (ie, education) or emotional support positively influenced recovery expectations, ${ }^{98} 101106$ negative emotions ${ }^{92} 98107108$ and risk appraisal on RTS. ${ }^{100} 109$ Social support was associated with greater adherence, ${ }^{526793104106}$ resilience, $^{9499}$ confidence $^{98108}$ and selfmotivation. ${ }^{106} 108$ Sources of social support changed throughout recovery with athletes reporting support from family, friends and teammates as important in early stages ${ }^{98} 110$ and a shift towards valuing support from coaches, physical therapists and other medical staff at later stages. ${ }^{5092} 110$ Furthermore, an injury role model, a forum to discuss and share experiences, and establishing new social roles and networks were important for staying motivated and effectively coping during recovery from injury. ${ }^{94} 96$ 99-101 103106108111112

\section{Engagement in care}

Injured athletes valued healthcare providers who engaged and involved them in their care through strategies such as goal setting. ${ }^{93} 97102105106$ A strong therapeutic alliance, where individual goals and values of athletes were respected led to positive rehabilitation experiences and improved trust in healthcare providers. $^{93} 96101102108$ Open discussions with healthcare providers and coaches ${ }^{92}$ allowed for athletes to have autonomy over surgery and RTS decisions and favour successful RTS.9 ${ }^{98} 103106$

\section{Contextual domain themes}

\section{Environmental influences}

An environment that promoted autonomy-supported behaviours were associated with greater levels of adherence to rehabilitation, independence and self-motivation. ${ }^{69} 70110$ Considerations important for adherence to rehabilitation included addressing situational factors (eg, lack of time or equipment, personal activity preferences) ${ }^{104}$ and making rehabilitation enjoyable or challenging. ${ }^{93} 97104$

\section{Sport culture}

A paucity of studies investigated the role of sport culture on recovery from a traumatic sport-related knee injury, but a few studies reported on the hyper-masculine culture of sport that validated enduring pain and downplaying injuries. ${ }^{92} 107$ Intense social pressure from peers ${ }^{106}$ and culture of risk-taking within sport ${ }^{92109}$ contributed to athletes considering premature RTS. ${ }^{106} 109$

\section{Cross-cutting concept of Individualisation}

Psychological, social or contextual factors manifested differently across athletes, highlighting a concept of 'individualisation'. For example, fear or anxiety may manifest as fear of re-injury/re-injury anxiety, ${ }^{657173747678829495105106113114}$ fear/anxiety of poor performance, ${ }^{92} 101106$ fear of failure, ${ }^{110}$ fear of the unknown or uncertainty of the situation (eg, contact sport, future long-term consequences) ${ }^{100109113}$ or fear of having to repeat rehabilitation. ${ }^{106} 108109113$ Similarly, individual coping strategies, ${ }^{83} 101111$ desire for social support, ${ }^{50112}$ preference to remain or avoid social roles ${ }^{96102105106}$ and environmental factors that influence treatment adherence exist. ${ }^{44104}$ Recovery expectations differed by gender and age, with men and younger athletes reporting greater expectations to RTS 557279113114 and different pressures to RTS (ie, peer pressure, performing through pain) compared with women or adults. ${ }^{106} 107109$

\section{Quality assessment}

MMAT ratings of included studies are summarised in online supplementary file 4. Most quantitative studies were rated as poor to moderate quality with only six studies rated as high quality (scoring four or higher). Qualitative studies had higher MMAT scores with nine studies rated as high quality. The highest level of evidence demonstrated by quantitative studies using the OCEBM model was level 2b (low-quality RCTs), with the majority classified as level 4 (61\%).

\section{DISCUSSION}

This comprehensive synthesis highlights a broad spectrum of psychological, social and contextual factors that mediate recovery after a traumatic time-loss sport-related knee injury. Athletes who have suffered a sport-related knee injury experience fear/anxiety as well as other barriers to progress recovery, most predominately at RTS. Across all recovery stages, athletes valued having an active role in their recovery and engaging in decision-making, as well as having their autonomy respected. Working with healthcare providers to set realistic expectations and receiving educational resources and social support was essential to successfully recover from a sport-related knee injury. The psychological, social and contextual factors were dynamic over recovery stages, and must be assessed and managed on an individual level.

\section{Psychological considerations for recovery from sport-related knee injury}

We identified four themes within the psychological domain: barriers to progress, active coping, independence and recovery expectations. Consistent with previous research, ${ }^{13}{ }^{14}$ fear is common in this population and consistently reported as a barrier for RTS. ${ }^{737678} 113$ However, other barriers such as frustration, anxiety, lack of confidence, ${ }^{92} 94114$ motivation $^{5458}$ or psychological readiness for RTS 4864658485 are also important. The desire for 'active coping' and 'independence' is consistent with the wellestablished self-determination theory ${ }^{115}$ that highlights satisfying individual needs for competence, relatedness and autonomy enhance recovery outcomes. ${ }^{116}$ Active coping strategies appear to be a common approach for athletes, ${ }^{117}$ which can promote motivation and resilience, particularly when facing a significant time-loss injury. ${ }^{5999101102110111}$ Athletes should be active in the development of their rehabilitation plan to foster autonomy and enhance internal locus of control. ${ }^{115}$ Given the importance of RTS for this population, early discussions and setting realistic expectations are vital to minimise negative emotions in later stages of recovery. ${ }^{9798} 112$

\section{Social considerations for recovery from sport-related knee injury}

Social support and engagement in care were the two themes identified in the social domain. As highlighted by others, ${ }^{20118}$ needs for social support change over time and continued re-evaluation of these needs are required to ensure the amount and type of social support desired by the athletes recovering from a knee injury is provided. Social support in the form of education was desired throughout recovery. Knowledge is a cornerstone of 
Table 5 Clinical implications

\begin{tabular}{ll}
\hline Key findings from review & Clinical implications \\
\hline $\begin{array}{l}\text { 1. Acknowledge the importance of psychological, } \\
\text { social and contextual factors }\end{array}$ & $\begin{array}{l}\text { Psychological, social and contextual factors should be acknowledged and not be viewed as less important than physical } \\
\text { factors. Given the wide range of psychological, social and contextual factors present, a holistic approach may more } \\
\text { adequately determine the individual needs }\end{array}$ \\
$\begin{array}{l}\text { 2. Psychological, social and contextual factors are } \\
\text { dynamic and change over time }\end{array}$ & $\begin{array}{l}\text { Psychological, social and contextual factors should be assessed early and monitored throughout recovery stages as they } \\
\text { change from the acute to RTS stage of recovery }\end{array}$ \\
$\begin{array}{l}\text { 3. Consider addressing social and contextual } \\
\text { factors to optimise care }\end{array}$ & $\begin{array}{l}\text { Strategies to develop a strong therapeutic alliance and engaging athletes in their care while considering how broader } \\
\text { environmental and societal factors impact decision-making should be vital components of recovery }\end{array}$ \\
4. Focus on the individual & $\begin{array}{l}\text { Interpretation and experience of psychological, social and contextual factors during recovery is individual, and will facilitate } \\
\text { the development of a recovery plan that best addresses individual needs and most likely to achieve success. }\end{array}$ \\
\hline
\end{tabular}

patient empowerment ${ }^{119}$ and may be an important consideration for coping with a significant time-loss injury.

Patient and healthcare provider engagement in care is drawing considerable attention, given its positive association with selfcare (eg, fostering autonomy) and overall health outcomes. ${ }^{120}$ Engagement is a necessary condition for a strong therapeutic relationship in physical therapy ${ }^{121}$ and a key component of a shared decision-making model. ${ }^{122}$ A strong therapeutic alliance was associated with improved rehabilitation outcomes in chronic musculoskeletal conditions ${ }^{123}$ and is likely relevant to recovery from a sport-related knee injury. Given the high risk of re-injury $^{124}$ and future PTOA $^{5}$ in this population, employing a shared decision-making approach early in the recovery process might enhance patient autonomy and the ability to make informed decisions regarding their current and future knee health. Although this model has been widely promoted as the ideal approach for RTS decisions, ${ }^{25}$ there appears to be a lack of understanding of shared decision-making in healthcare. ${ }^{125}$

\section{Contextual considerations for recovery from sport-related knee injury}

Contextual factors during recovery from a sport-related knee injury are rarely studied, but consistent themes of environmental influences and sport culture were present. Our findings align with prior research that highlight sport cultivates a culture of 'no pain no gain' and risk-taking, which can contribute to premature RTS and further injury. ${ }^{31}$ While it is not typical for healthcare providers to consider the systemic environmental factors that shape an athlete's recovery from injury, it is essential to understand that this context may be a stronger determinant of recovery than the treatment provided as it determines access to resources and shapes behaviours.

\section{Individual considerations for recovery from sport-related knee injury}

The importance of tailoring recovery to the individual is essential if patient-centred care is the goal. ${ }^{126}$ Understanding personal goals, values and definitions of success are important as they influence recovery expectations. ${ }^{127}$ It is clear that individual characteristics such as gender, age and level of sport participation influence the presentation of psychological, social and contextual factors across recovery stages and can shape recovery outcomes, such as sport participation after an injury. For example, prior studies have reported that men are more likely to return to their pre-injury sport compared with women after ACLR. ${ }^{3}$ This might reflect gender-specific and sex-specific psychological, social and contextual factors. Specifically, men may embrace the hyper-masculine sport culture and indulge in risky behaviours ${ }^{92} 107$ than women who may be more risk adverse. ${ }^{109}$ Similarly, the social peer pressure to RTS may be more apparent during adolescence $e^{106}$ as sport participation is an important form of social connection during the developmental years. ${ }^{128}$ It is likely that cultural perceptions, religion, social gradient, and multiple other social and contextual factors also influence recovery and drive specific actions and behaviours in different populations. A better understanding of an individual's context is essential to developing a holistic approach to optimise recovery.

\section{Clinical implications}

Table 5 provides the summary of the clinical implications of our findings.

\section{Strengths and limitations}

The broad research question and inclusion of diverse study designs that contained both quantitative and qualitative data resulted in a rich, in-depth mapping of the current evidencebase. Using two established frameworks required us to consider factors and themes in psychological, social and contextual domains, and provided more clarity about the types of factors and themes that are present in this population. We recognise that many identified factors share overlapping domains; however, this complexity and interrelatedness is critical to an accurate representation and understanding of psychological, social and contextual factors across recovery stages.

Scoping reviews rarely evaluate the methodological quality of included studies, but we chose to take this additional step to confidently develop our conclusions and identify the knowledge gaps. ${ }^{29}$ It is important to reiterate that most included studies were of poor to moderate methodological quality, indicating the need for higher-quality studies in this field. With that said, the consistent themes that emerged across studies provide us with confidence in our conclusions.

Despite a comprehensive search strategy, most included studies were limited to ACL injuries, pointing to a paucity of evidence related to other traumatic time-loss lower extremity injuries potentially limiting the generalisability of our findings. With that said, it is possible that our findings and their clinical implications may have broad applicability across recovery from other traumatic sport injuries. The studies contributing data to the RTS stage were heterogeneous in time since injury as many were retrospective in nature. While we followed a rigorous approach and engaged a research team with diverse expertise, formal consultation with other stakeholders (ie, persons with traumatic knee injuries), disciplines (ie, social scientists) and inclusion of grey literature may have provided further insight at all stages of the review.

\section{Future directions}

This review provides a foundation to understand the role and interactions between psychological, social and contextual factors 


\section{What are the new findings?}

- Psychological, social and contextual domains play an important role in recovery following a sport-related knee injury.

- Athletes who suffer a sport-related time-loss knee injury experience many barriers to progress beyond fear across all stages of recovery.

- Athletes value having an active role in their recovery, being engaged in decision-making, and having their autonomy respected.

- Psychological, social and contextual factors change over time and should be assessed early and often throughout recovery stages.

- Individual consideration of psychological, social and contextual factors is an essential component of an evidencebased approach to management of sport-related knee injuries.

\section{How might it impact on clinical practice in the future?}

- Recovery from a traumatic time-loss knee injury does not always have a happy ending, with a significant proportion of individuals failing to return to pre-injury sport and reporting reduced health-related quality of life.

- Despite many reports about the importance of considering psychological, social and contextual factors after a sportrelated knee injury, management tends to prioritise physical impairments.

- Assessment and monitoring of psychological, social and contextual factors should be conducted throughout recovery, and not just at the return to sport stage

- Consider an individual and patient-centered focus when managing psychological, social and contextual factors to optimise recovery

as it relates to recovery following a traumatic time-loss sportrelated knee injury. Although a great deal is known about interpersonal psychological factors, particularly fear, further research is needed to understand social and contextual factors, including the broader influence of systemic determinants on recovery from a traumatic time-loss sport-related knee injury. Overall, there is a paucity of knowledge about the status of psychological, social and contextual factors at the time of injury. Better understanding of these factors in the early stages of recovery will assist in the development of screening strategies for poor recovery and facilitate research aimed at understanding how these factors change and are influenced over time.

There is an opportunity to address inconsistencies in terminology used to define social and contextual factors within the sport and exercise medicine/rehabilitation field, and align with broader definitions from the social sciences. Furthermore, developing consensus on how individual psychological, social and contextual factors are defined and reported is essential for amalgamating data across studies and will promote uptake of these constructs in clinical practice.

Currently, there is a tendency in the sport and exercise medicine/rehabilitation field to lump psychological and social factors together and to focus care at the individual level. To date, few studies have considered the broader systemic aspects of social (societal) and contextual conditions (eg, social isolation or social gradient) in which athletes are injured and recover. ${ }^{19} \mathrm{~A}$ better understanding of how these broader social and contextual factors shape an athletes recovery is essential to optimising that process and improving overall well-being.

\section{CONCLUSIONS}

This scoping review highlighted the broad spectrum of psychological, social and contextual factors that can play a role during the acute, rehabilitation and RTS stages of recovery following a traumatic time-loss sport-related knee injury. The experience and interpretation of these factors are individual. While high-quality research is needed in this field, there appears to be consistent evidence of the impact of psychological and social factors on recovery. Individualised consideration of these factors should be an essential component of an evidence-based approach to managing sport-related knee injuries.

Twitter Linda K Truong @LKTphysio, Christopher J Holt @choltpt, Christina Y Le @yegphysio, Maxi Miciak @twitter.com/MaxiMiciak and Jackie LWhittaker @jwhittak_physio

Acknowledgements The authors would like to acknowledge the assistance of $\mathrm{Dr}$ Linda Li who provided feedback on the first draft of the manuscript and Maria Tan, Health Science Librarian, who contributed to the development of the search strategy. LK Truong was supported by a First Year Recruitment Award provided by the Faculty of Rehabilitation Medicine, University of Alberta.

Collaborators Linda C Li; Maria Tan.

Contributors LKT and JLW were responsible for the conception of the study and along with Maria Tan developed the search strategy. LKT executed the search strategy. All authors independently reviewed records, assessed the methodological quality of included studies and extracted data. LKT was responsible for the first draft of the manuscript. All authors contributed to the interpretation of the findings, critical revision of the manuscript and reviewed the document prior to submission.

Funding The authors have not declared a specific grant for this research from any funding agency in the public, commercial or not-for-profit sectors.

Competing interests None declared.

Patient consent for publication Not required.

Provenance and peer review Not commissioned; externally peer reviewed.

Open access This is an open access article distributed in accordance with the Creative Commons Attribution Non Commercial (CC BY-NC 4.0) license, which permits others to distribute, remix, adapt, build upon this work non-commercially, and license their derivative works on different terms, provided the original work is properly cited, appropriate credit is given, any changes made indicated, and the use is non-commercial. See: http://creativecommons.org/licenses/by-nc/4.0/.

\section{ORCID iDs}

Linda K Truong http://orcid.org/0000-0002-0728-6297

Amber D Mosewich http://orcid.org/0000-0001-5870-4738

Christopher J Holt http://orcid.org/0000-0003-4381-3661

Christina Y Le http://orcid.org/0000-0003-0241-5157

Maxi Miciak http://orcid.org/0000-0003-4686-9206

Jackie L Whittaker http://orcid.org/0000-0002-6591-4976

\section{REFERENCES}

1 Emery CA, Meeuwisse WH, McAllister JR. Survey of sport participation and sport injury in Calgary and area high schools. Clin J Sport Med 2006;16:20-6.

2 Finch CF, Kemp JL, Clapperton AJ. The incidence and burden of hospital-treated sports-related injury in people aged 15+ years in Victoria, Australia, 2004-2010: a future epidemic of osteoarthritis? Osteoarthritis Cartilage 2015;23:1138-43.

3 Ardern CL, Taylor NF, Feller JA, et al. Fifty-five per cent return to competitive sport following anterior cruciate ligament reconstruction surgery: an updated systematic review and meta-analysis including aspects of physical functioning and contextual factors. Br J Sports Med 2014;48:1543-52.

4 Swart NM, van Oudenaarde K, Bierma-Zeinstra SMA, et al. Predicting no return to sports after three months in patients with traumatic knee complaints in general practice by combining patient characteristics, trauma characteristics and knee complaints. Eur J Gen Pract 2019;25:205-13.

5 Lie MM, Risberg MA, Storheim K, et al. What's the rate of knee osteoarthritis 10 years after anterior cruciate ligament injury? An updated systematic review. $\mathrm{Br}$ J Sports Med 2019;53:1162-7. 
6 Whittaker JL, Toomey CM, Woodhouse LJ, et al. Association between MRI-defined osteoarthritis, pain, function and strength 3-10 years following knee joint injury in youth sport. Br J Sports Med 2018;52:934-9.

7 Stein T, Mehling AP, Welsch F, et al. Long-Term outcome after arthroscopic meniscal repair versus arthroscopic partial meniscectomy for traumatic meniscal tears. Am J Sports Med 2010;38:1542-8.

8 Ardern CL, Taylor NF, Feller JA, et al. Return-to-sport outcomes at 2 to 7 years after anterior cruciate ligament reconstruction surgery. Am J Sports Med 2012:40:41-8

9 Cole BJ, Cotter EJ, Wang KC, et al. Patient understanding, expectations, outcomes, and satisfaction regarding anterior cruciate ligament injuries and surgical management. Arthroscopy 2017;33:1092-6.

10 Filbay SR, Ackerman IN, Russell TG, et al. Health-Related quality of life after anterior cruciate ligament reconstruction: a systematic review. Am J Sports Med 2014:42:1247-55.

11 Brewer B, Andersen MB, Van Raalte JL. Psychological aspects of sport injury rehabilitation: Toward a biopsychosocial approach. In: Mostofsky DL, Zaichkowsky LD, eds. Medical and psychological aspects of sport and exercise, 2002: 41-54.

12 Wiese-bjornstal DM, Smith AM, Shaffer SM, et al. An integrated model of response to sport injury: psychological and sociological dynamics. J App/ Sport Psychol 1998; 10:46-69.

13 Forsdyke D, Smith A, Jones M, et al. Psychosocial factors associated with outcomes of sports injury rehabilitation in competitive athletes: a mixed studies systematic review. Br J Sports Med 2016;50:537-44.

14 Ardern CL, Taylor NF, Feller JA, et al. A systematic review of the psychological factors associated with returning to sport following injury. Br J Sports Med 2013;47:1120-6.

15 te Wierike SCM, van der Sluis A, van den Akker-Scheek I, Akker-Scheek vanden I, et al. Psychosocial factors influencing the recovery of athletes with anterior cruciate ligament injury: a systematic review. Scand J Med Sci Sports 2013;23:527-40

16 Bandura A. Self-Efficacy: toward a unifying theory of behavioral change. Advances in Behaviour Research and Therapy 1978;1:139-61.

17 Everhart JS, Best TM, Flanigan DC. Psychological predictors of anterior cruciate ligament reconstruction outcomes: a systematic review. Knee Surg Sports Traumatol Arthrosc 2015;23:752-62

18 Bianco T, Eklund RC. Conceptual considerations for social support research in sport and exercise settings: the case of sport injury. J Sport Exerc Psychol 2001:23:85-107.

19 Marmot M, Wilkinson R. Social determinants of health. 2nd. Oxford: Oxford University Press, 2005

20 Bianco T. Social support and recovery from sport injury: elite skiers share their experiences. Res Q Exerc Sport 2001;72:376-88.

21 Babatunde F, MacDermid J, MacIntyre N. Characteristics of therapeutic alliance in musculoskeletal physiotherapy and occupational therapy practice: a scoping review of the literature. BMC Health Serv Res 2017;17:375.

22 World Health Organization. Towards a common language for functioning, disability and health: the International classification of functioning, disability, and health, 2002.

23 Orhan C, Van Looveren E, Cagnie B, et al. Are pain beliefs, cognitions, and behaviors influenced by race, ethnicity, and culture in patients with chronic musculoskeletal pain: a systematic review. Pain Physician 2018;21:541-558.

24 Main CJ, Foster N, Buchbinder R. How important are back pain beliefs and expectations for satisfactory recovery from back pain? Best Pract Res Clin Rheumatol 2010;24:205-17.

25 Ardern CL, Glasgow P, Schneiders A, et al. 2016 consensus statement on return to sport from the first world Congress in sports physical therapy, Bern. Br J Sports Med 2016;50:853-64

26 Munn Z, Peters MDJ, Stern C, et al. Systematic review or scoping review? guidance for authors when choosing between a systematic or scoping review approach. BMC Med Res Methodol 2018;18:143.

27 Arksey H, O'Malley L. Scoping studies: towards a methodological framework. Int J Soc Res Methodol 2005:8:19-32.

28 Joanna Briggs Institute reviewers' manual. Methodology for JBI scoping reviews. In: The Joanna Briggs Institute, 2015

29 Levac D, Colquhoun H, O'Brien KK. Scoping studies: advancing the methodology. Implement Sci 2010;5:69.

30 Tricco AC, Lillie E, Zarin W, et al. Prisma extension for scoping reviews (prisma-scr): checklist and explanation. Ann Intern Med 2018;169:467.

31 Wiese-Bjornstal DM. Psychology and socioculture affect injury risk, response, and recovery in high-intensity athletes: a consensus statement. Scand J Med Sci Sports 2010;20:103-11.

32 Brewer B, Redmond, Charles JR. Psychology of sport injury rehabilitation. Champaign, IL: Human Kinetics, 2017.

33 Braun V, Clarke V. Using thematic analysis in psychology. Qual Res Psychol 2006:3:77-101.

34 Testa MA, Simonson DC. Assessment of quality-of-life outcomes. N Eng/ J Med 1996;334:835-40.
35 Hong QN, Fàbregues S, Bartlett G, et al. The mixed methods appraisal tool (MMAT) version 2018 for information professionals and researchers. Education for Information 2018;34:285-91.

36 Souto RQ, Khanassov V, Hong QN, et al. Systematic mixed studies reviews: updating results on the reliability and efficiency of the mixed methods appraisal tool. Int J Nurs Stud 2015:52:500-1.

37 Pace R, Pluye P, Bartlett G, et al. Testing the reliability and efficiency of the pilot mixed methods appraisal tool (MMAT) for systematic mixed studies review. Int $J$ Nurs Stud 2012;49:47-53

38 Mainwaring LM, Hutchison M, Bisschop SM, et al. Emotional response to sport concussion compared to ACL injury. Brain Injury 2010;24:589-97.

39 Ardern CL, Taylor NF, Feller JA, et al. Psychological responses matter in returning to preinjury level of sport after anterior cruciate ligament reconstruction surgery. Am J Sports Med 2013;41:1549-58.

40 Baranoff J, Hanrahan SJ, Connor JP. The roles of acceptance and catastrophizing in rehabilitation following anterior cruciate ligament reconstruction. J Sci Med Sport 2015; 18:250-4.

41 Brewer BW, Cornelius AE, Sklar JH, et al. Pain and negative mood during rehabilitation after anterior cruciate ligament reconstruction: a daily process analysis. Scand J Med Sci Sports 2007;17:520-9.

42 Brewer BW, Cornelius AE, Van Raalte JL, et al. Attributions for recovery and adherence to rehabilitation following anterior cruciate ligament reconstruction: a prospective analysis. Psychol Health 2000;15:283-91.

43 Brewer BW, Cornelius AE, Stephan Y, et al. Self-protective changes in athletic identity following anterior cruciate ligament reconstruction. Psychol Sport Exerc 2010;11:1-5.

44 Brewer BW, Cornelius AE, Van Raalte JL, et al. Predictors of adherence to home rehabilitation exercises following anterior cruciate ligament reconstruction. Rehabil Psychol 2013;58:64-72

45 Brewer BW, Cornelius AE, Van Raalte JL, et al. Adversarial growth after anterior cruciate ligament reconstruction. J Sport Exerc Psychol 2017;39:134-44.

46 Chmielewski TL, Zeppieri G, Lentz TA, et al. Longitudinal changes in psychosocial factors and their association with knee pain and function after anterior cruciate ligament reconstruction. Phys Ther 2011;91:1355-66.

47 Gignac MAM, Cao X, Ramanathan S, et al. Perceived personal importance of exercise and fears of re-injury: a longitudinal study of psychological factors related to activity after anterior cruciate ligament reconstruction. BMC Sports Sci Med Rehabil 2015;7:4

48 Langford JL, Webster KE, Feller JA. A prospective longitudinal study to assess psychological changes following anterior cruciate ligament reconstruction surgery. $\mathrm{Br}$ J Sports Med 2009:43:377-8.

49 Lepley AS, Pietrosimone B, Cormier ML. Quadriceps function, knee pain, and selfreported outcomes in patients with anterior cruciate ligament reconstruction. J Athl Train 2018:53:337-46.

50 Masten R, Stražar K, Žilavec I, et al. Psychological response of athletes to injury. Kinesiology 2014;46:127-34.

51 Morrey MA, Stuart MJ, Smith AM, et al. A longitudinal examination of athletes' emotional and cognitive responses to anterior cruciate ligament injury. Clin J Sport Med 1999:9:63-9.

52 Niven A, Nevill A, Sayers F, et al. Predictors of rehabilitation intention and behavior following anterior cruciate ligament surgery: an application of the theory of planned behavior. Scand J Med Sci Sports 2012;22:316-22.

53 Paterno MV, Flynn K, Thomas S, et al. Self-Reported fear predicts functional performance and second $A C L$ injury after $A C L$ reconstruction and return to sport: a pilot study. Sports Health 2018;10:228-33.

54 Roessler KK, Andersen TE, Lohmander S, et al. Motives for sports participation as predictions of self-reported outcomes after anterior cruciate ligament injury of the knee. Scand J Med Sci Sports 2015;25:435-40.

55 Rosenberger PH, Jokl P, Cameron A, et al. Shared decision making, preoperative expectations, and postoperative reality: differences in physician and patient predictions and ratings of knee surgery outcomes. Arthroscopy 2005;21:562-9.

56 Scherzer CB, Brewer BW, Cornelius AE, et al. Psychological skills and adherence to rehabilitation after reconstruction of the anterior cruciate ligament. J Sport Rehabil 2001;10:165-72.

57 Shapiro JL, Brewer BW, Cornelius AE, et al. Patterns of emotional response to $\mathrm{ACL}$ reconstruction surgery. J Clin Sport Psychol 2017;11:169-80.

58 Sonesson S, Kvist J, Ardern C, et al. Psychological factors are important to return to pre-injury sport activity after anterior cruciate ligament reconstruction: expect and motivate to satisfy. Knee Surg Sports Traumatol Arthrosc 2017;25:1375-84.

59 Thomeé P, Währborg P, Börjesson M, et al. Self-Efficacy, symptoms and physical activity in patients with an anterior cruciate ligament injury: a prospective study. Scand J Med Sci Sports 2007;17:238-45.

60 Thomeé $P$, Währborg P, Börjesson M, et al. Self-efficacy of knee function as a preoperative predictor of outcome 1 year after anterior cruciate ligament reconstruction. Knee Surg Sports Traumatol Arthr 2008;16:118-27.

61 Lentz TA, Zeppieri G, George SZ, et al. Comparison of physical impairment, functional, and psychosocial measures based on fear of reinjury/lack of confidence and return-to-sport status after ACL reconstruction. Am J Sports Med 2015;43:345-53. 
62 Nwachukwu BU, Chang B, Voleti PB, et al. Preoperative short form health survey score is predictive of return to play and minimal clinically important difference at a minimum 2-year follow-up after anterior cruciate ligament reconstruction. Am J Sports Med 2017:45:2784-90.

63 McAllister DR, Tsai AM, Dragoo JL, et al. Knee function after anterior cruciate ligament injury in elite collegiate athletes. Am J Sports Med 2003;31:560-3.

64 Ardern CL, Österberg A, Tagesson S, et al. The impact of psychological readiness to return to sport and recreational activities after anterior cruciate ligament reconstruction. Br J Sports Med 2014;48:1613-9.

65 Ardern CL, Taylor NF, Feller JA, et al. Fear of re-injury in people who have returned to sport following anterior cruciate ligament reconstruction surgery. J Sci Med Sport 2012;15:488-95.

66 Bennell KL, van Ginckel A, Kean CO, et al. Patient knowledge and beliefs about knee osteoarthritis after anterior cruciate ligament injury and reconstruction. Arthritis Care Res 2016:68:1180-5

67 Brewer BW, Cornelius AE, Van Raalte JL, et al. Age-Related differences in predictors of adherence to rehabilitation after anterior cruciate ligament reconstruction. J Athl Train 2003;38:158-62.

68 Brewer BW, Cornelius AE, Van Raalte JL, et al. Presurgical anxiety, concerns, and anticipated benefits associated with anterior cruciate ligament reconstruction. Hong Kong Journal of Sports Medicine \& Sports Science 2002;15:1-8.

69 Chan DK, Lonsdale C, Ho PY, et al. Patient motivation and adherence to postsurgery rehabilitation exercise recommendations: the influence of physiotherapists' autonomy-supportive behaviors. Arch Phys Med Rehabil 2009;90:1977-82.

70 Chan DK-C, Hagger MS, Spray CM. Treatment motivation for rehabilitation after a sport injury: application of the trans-contextual model. Psychol Sport Exerc 2011;12:83-92.

71 Chmielewski TL, Jones D, Day T, et al. The association of pain and fear of movement/ reinjury with function during anterior cruciate ligament reconstruction rehabilitation. J Orthop Sports Phys Ther 2008;38:746-53.

72 Feucht MJ, Cotic M, Saier T, et al. Patient expectations of primary and revision anterior cruciate ligament reconstruction. Knee Surg Sports Traumatol Arthrosc 2016;24:201-7.

73 Flanigan DC, Everhart JS, Pedroza A, et al. Fear of reinjury (kinesiophobia) and persistent knee symptoms are common factors for lack of return to sport after anterior cruciate ligament reconstruction. Arthroscopy 2013;29:1322-9.

74 George SZ, Lentz TA, Zeppieri G, et al. Analysis of shortened versions of the Tampa scale for Kinesiophobia and pain Catastrophizing scale for patients after anterior cruciate ligament reconstruction. Clin J Pain 2012;28:73-80.

75 Hart HF, Collins NJ, Ackland DC, et al. Is impaired knee confidence related to worse kinesiophobia, symptoms, and physical function in people with knee osteoarthritis after anterior cruciate ligament reconstruction? I Sci Med Sport 2015;18:512-7.

76 Kvist J, Ek A, Sporrstedt K, et al. Fear of re-injury: a hindrance for returning to sports after anterior cruciate ligament reconstruction. Knee Surg Sports Traumatol Arthrosc 2005:13:393-7.

77 Lam KC, Thomas SS, Valier ARS, et al. Previous knee injury and health-related quality of life in collegiate athletes. J Ath/ Train 2017;52:534-40.

78 Lentz TA, Zeppieri G, Tillman SM, et al. Return to preinjury sports participation following anterior cruciate ligament reconstruction: contributions of demographic, knee impairment, and self-report measures. J Orthop Sports Phys Ther 2012;42:893-901.

79 Mancuso CA, Sculco TP, Wickiewicz TL, et al. Patients' expectations of knee surgery. J Bone Joint Surg Am 2001;83:1005-12.

80 McGuine TA, Winterstein A, Carr K, et al. Changes in self-reported knee function and health-related quality of life after knee injury in female athletes. Clin J Sport Med 2012;22:334-40.

81 Thomeé $\mathrm{P}$, Währborg $\mathrm{P}$, Börjesson $\mathrm{M}$, et al. Determinants of self-efficacy in the rehabilitation of patients with anterior cruciate ligament injury. J Rehabil Med 2007;39:486-92.

82 Tripp DA, Stanish W, Ebel-Lam A, et al. Fear of reinjury, negative affect, and catastrophizing predicting return to sport in recreational athletes with anterior cruciate ligament injuries at 1 year postsurgery. Sport Exerc Perform Psychol 2011;1:38-48.

83 Udry E, Donald Shelbourne K, Gray T. Psychological readiness for anterior cruciate ligament surgery: describing and comparing the adolescent and adult experiences. J Athl Train 2003;38:167-71.

84 Webster KE, Feller JA, Lambros C. Development and preliminary validation of a scale to measure the psychological impact of returning to sport following anterior cruciate ligament reconstruction surgery. Phys Ther Sport 2008;9:9-15.

85 Ardern CL, Taylor NF, Feller JA, et al. Sports participation 2 years after anterior cruciate ligament reconstruction in athletes who had not returned to sport at 1 year: a prospective follow-up of physical function and psychological factors in 122 athletes. Am J Sports Med 2015;43:848-56.

86 Mankad A, Gordon S, Wallman K. Psycholinguistic analysis of emotional disclosure: a case study in sport injury. J Clin Sport Psychol 2009;3:182-96.

87 Samuel RD, Tenenbaum G, Mangel E, et al. Athletes' experiences of severe injuries as a career-change event. J Sport Psychol Action 2015;6:99-120.
88 Tagesson S, Kvist J. Greater fear of re-injury and increased tibial translation in patients who later sustain an $A C L$ graft rupture or a contralateral $A C L$ rupture: a pilot study. J Sports Sci 2016;34:125-32.

89 Cupal DD, Brewer BW. Effects of relaxation and guided imagery on knee strength, reinjury anxiety, and pain following anterior cruciate ligament reconstruction. Rehabil Psychol 2001;46:28-43.

90 Maddison R, Prapavessis H, Clatworthy M, et al. Guided imagery to improve functional outcomes post-anterior cruciate ligament repair: randomized-controlled pilot trial. Scand J Med Sci Sports 2012;22:816-21.

91 Thomeé $\mathrm{P}$, Währborg $\mathrm{P}$, Börjesson $\mathrm{M}$, et al. A randomized, controlled study of a rehabilitation model to improve knee-function self-efficacy with $A C L$ injury. J Sport Rehabil 2010;19:200-13.

92 Carson F, Polman R. Experiences of professional rugby union players returning to competition following anterior cruciate ligament reconstruction. Physical Therapy in Sport 2012;13:35-40.

93 Evans L, Hardy L. Injury rehabilitation: a qualitative follow-up study. Res Q Exerc Sport 2002;73:320-9.

94 Ezzat AM, Brussoni M, Whittaker JL, et al. A qualitative investigation of the attitudes and beliefs about physical activity and post-traumatic osteoarthritis in young adults 3-10 years after an intra-articular knee injury. Phys Ther Sport 2018;32:98-108.

95 Filbay SR, Crossley KM, Ackerman IN. Activity preferences, lifestyle modifications and re-injury fears influence longer-term quality of life in people with knee symptoms following anterior cruciate ligament reconstruction: a qualitative study. J Physiother 2016:62:103-10.

96 Ford UW, Gordon S. Coping with sport injury: resource loss and the role of social support. J Pers Interpers Los 1999;4:243-56.

97 Heijne A, Axelsson K, Werner S, et al. Rehabilitation and recovery after anterior cruciate ligament reconstruction: patients' experiences. Scand J Med Sci Sports 2008; 18:325-35.

98 Ivarsson A, Stambulova N, Johnson U. Injury as a career transition: experiences of a Swedish elite handball player. Int J Sport Exerc Psychol 2016;16:1-17.

99 Johnson U, Ivarsson A, Karlsson J, et al. Rehabilitation after first-time anterior cruciate ligament injury and reconstruction in female football players: a study of resilience factors. BMC Sports Sci Med Rehabil 2016;8:20.

100 Johnston LH, Carroll D. The context of emotional responses to athletic injury: a qualitative analysis. J Sport Rehabil 1998;7:206-20.

101 Mainwaring LM. Restoration of self: a model for the psychological response of athletes to severe knee injuries. Can J Rehabil 1999;12:145-56.

102 Nordahl B, Sjöström R, Westin M, et al. Experiences of returning to elite alpine skiing after $A C L$ injury and $A C L$ reconstruction. Int J Adolesc Med Health 2014;26:69-77.

103 Olofsson L, Fjellman-Wiklund A, Söderman K. From loss towards restoration: experiences from anterior cruciate ligament injury. Adv Physiother 2010;12:50-7.

104 Pizzari T, McBurney H, Taylor NF, et al. Adherence to anterior cruciate ligament rehabilitation: a qualitative analysis. J Sport Rehabil 2002;11:90-102.

105 Podlog L, Eklund RC. A longitudinal investigation of competitive athletes' return to sport following serious injury. J App/ Sport Psychol 2006;18:44-68.

106 Podlog L, Wadey R, Stark A, et al. An adolescent perspective on injury recovery and the return to sport. Psychol Sport Exerc 2013;14:437-46.

107 Ronkainen NJ, Ryba TV. Is hockey just a game? contesting meanings of the ice hockey life projects through a career-threatening injury. J Sports Sci 2017;35:923-8.

108 Scott SM, Perry MA, Sole G. "Not always a straight path": patients' perspectives following anterior cruciate ligament rupture and reconstruction. Disabil Rehabil 2018:40:2311-7.

109 Thing LF. "Voices of the broken body." The resumption of non-professional female players' sports careers after anterior cruciate ligament injury. The female player's dilemma: is she willing to run the risk? Scand J Med Sci Sports 2006;16:364-75

110 Carson F, Polman RCJ. Acl injury rehabilitation: a psychological case study of a professional rugby Union player. J Clin Sport Psychol 2008;2:71-90.

111 Carson F, Polman RCJ. The facilitative nature of avoidance coping within sports injury rehabilitation. Scand J Med Sci Sports 2010;20:235-40.

112 Rock JA, Jones MV. A preliminary investigation into the use of counseling skills in support of rehabilitation from sport injury. J Sport Rehabil 2002;11:284-304.

113 Ross CA, Clifford A, Louw QA. Factors informing fear of reinjury after anterior cruciate ligament reconstruction. Physiother Theory Pract 2017;33:103-14.

114 Tjong VK, Murnaghan ML, Nyhof-Young JM, et al. A qualitative investigation of the decision to return to sport after anterior cruciate ligament reconstruction: to play or not to play. Am J Sports Med 2014;42:336-42.

115 Ryan RM, Deci EL. Self-determination theory and the facilitation of intrinsic motivation, social development, and well-being. Am Psychol 2000:55:68-78.

116 Podlog L, Dimmock J, Miller J. A review of return to sport concerns following injury rehabilitation: practitioner strategies for enhancing recovery outcomes. Phys Ther Sport 2011;12:36-42

117 Quinn AM, Fallon BJ. The changes in psychological characteristics and reactions of elite athletes from injury onset until full recovery. J App/ Sport Psychol 1999;11:210-29.

118 Johnston LH, Carroll D. The provision of social support to injured athletes: a qualitative analysis. J Sport Rehabil 1998;7:267-84 
119 Werbrouck A, Swinnen E, Kerckhofs E, et al. How to empower patients? A systematic review and meta-analysis. Trans/ Behav Med 2018;8:660-74.

120 Bright FAS, Kayes NM, Worrall L, et al. A conceptual review of engagement in healthcare and rehabilitation. Disabil Rehabil 2015;37:643-54.

121 Miciak M, Mayan M, Brown C, et al. The necessary conditions of engagement for the therapeutic relationship in physiotherapy: an interpretive description study. Arch Physiother 2018;8:3.

122 Légaré F, Ratté S, Stacey D, et al. Interventions for improving the adoption of shared decision making by healthcare professionals. Cochrane Database Syst Rev 2010;5:CD006732.

123 Kinney M, Seider J, Beaty AF, et al. The impact of therapeutic alliance in physical therapy for chronic musculoskeletal pain: a systematic review of the literature. Physiother Theory Pract 2018:6:1-13.

124 Wiggins AJ, Grandhi RK, Schneider DK, et al. Risk of secondary injury in younger athletes after anterior cruciate ligament reconstruction: a systematic review and meta-analysis. Am J Sports Med 2016;44:1861-76.

125 Légaré $F$, Thompson-Leduc $P$. Twelve myths about shared decision making. Patient Educ Couns 2014;96:281-6.

126 Bastemeijer CM, Voogt L, van Ewijk JP, et al. What do patient values and preferences mean? A taxonomy based on a systematic review of qualitative papers. Patient Educ Couns 2017:100:871-81.

127 Carroll LJ, Lis A, Weiser S, et al. How well do you expect to recover, and what does recovery mean, anyway? qualitative study of expectations after a musculoskeletal injury. Phys Ther 2016;96:797-807.
128 Neely K, Holt N. Positive youth development through sport: a review 2011:2:299-316.

129 Fuller CW, Molloy MG, Bagate C, et al. Consensus statement on injury definitions and data collection procedures for studies of injuries in rugby Union. Br J Sports Med 2007;41:328-31.

130 Swann C, Moran A, Piggott D. Defining elite athletes: issues in the study of expert performance in sport psychology. Psychol Sport Exerc 2015;16:3-14.

131 Walker N, Thatcher J, Lavallee D. A preliminary development of the Re-Injury anxiety inventory (RIAI). Phys Ther Sport 2010;11:23-9.

132 Vealey RS, Chase MA. Advances in sport psychology. In: Self-confidence in sport. 3rd edn. Champaign, IL, US: Human Kinetics, 2008: 430-5.

133 Lazarus R, Folkman S. Stress, appraisal, and coping. New York: Springer Pub. Co, 1984.

134 Stryker S, Burke PJ. The past, present, and future of an identity theory. Soc Psychol Q 2000;63:284-97.

135 Fletcher D, Sarkar M. Mental fortitude training: an evidence-based approach to developing psychological resilience for sustained success. I Sport Psychol Action 2016;7:135-57

136 Smith AD. Perception and belief. Philos Phenomenol Res 2001;62:283-309.

137 Rees T, Hardy L, Evans L. Construct validity of the social support survey in sport. Psychol Sport Exerc 2007;8:355-68.

138 Bordin ES. The generalizability of the psychoanalytic concept of the working alliance. Psychotherapy: Theory, Research \& Practice 1979;16:252-60.

139 Richardson SOA, B; Morris M. T Overtraining athletes: personal journeys in sport. Champaign, IL: Human Kinetics, 2008. 